Editorial Office Notes:

RES-07-678.R2

Original Article

Received: 5 December 2007

Invited to revise: 2 January 2008, 11 February 2008

Revised: 28 January 2008, 12 February 2008

Accepted: 21 February 2008

Associate Editor: David Hui

Editor in chief sign off date: Phil Bardin 23 May 2008 


\section{Revision of the severity rating and classification of hospital-acquired pneumonia in the Japanese Respiratory Society guidelines}

Masafumi Seki, ${ }^{1}$ Akira Watanabe, ${ }^{2}$ Keiichi Mikasa, ${ }^{3}$ Junichi Kadota ${ }^{4}$ and Shigeru Kohno ${ }^{1}$

${ }^{1}$ Department of Molecular Microbiology and Immunology, Nagasaki University Graduate School of Medical Sciences, Nagasaki, ${ }^{2}$ Research Division for Development of Anti-Infective Agents, Institute of Development, Aging and Cancer, Tohoku University, Sendai, ${ }^{3}$ Center for Infectious Diseases, Nara Medical University, Nara, ${ }^{4}$ Second Department of Internal Medicine, Oita University Faculty of Medicine, Oita, Japan.

Short title: New criteria for severity of HAP

Correspondence: Masafumi Seki, MD, $\mathrm{PhD}$

$2^{\text {nd }}$ Department of Internal Medicine, Nagasaki University Hospital, Sakamoto 1-7-1, Nagasaki, 852-8501, Japan. Tel: +81-95-849-7273, Fax: 095-849-7285

E-mail: seki@nagasaki-u.ac.jp 


\section{ABSTRACT}

Background and objective: Based on the results of a multicentre collaborative survey of hospital-acquired pneumonia (HAP) conducted in Japan, the severity rating and classification of pneumonia in the Japanese Respiratory Society guidelines for management of HAP were examined.

Methods: Parameters for the severity classification were selected from the factors associated with prognosis in the HAP survey and in other previous reports. Depending on the presence of the parameters listed below, patients with HAP were stratified into those with high, moderate, or low-risk. The high-risk group was defined as patients with three or more of the following risk factors: "malignant tumour or immunocompromised status", "impaired consciousness", "requiring fraction of inspired oxygen $\left(\mathrm{FiO}_{2}\right)>35 \%$ to maintain $\mathrm{SaO}_{2}>90 \%$ ", "male aged 70 years or older, or female aged 75 years or older" and "oliguria or dehydration." The moderate-risk group was defined as patients with any of the secondary risk factors as follows: "C-reactive protein $\geq 200 \mathrm{mg} / \mathrm{L}$ " and "extent of infiltration on CXR covers at least $2 / 3$ of one lung". The low-risk group was defined as all other patients.

Results: Application of this classification scheme to the patients enrolled in the HAP survey revealed a mortality rate of $40.8 \%(98 / 240)$ in the high-risk group, which was significantly higher than the mortality rates in the moderate and low-risk groups: $24.9 \%(69 / 277)$ and $12.1 \%(101 / 834)$, respectively.

Conclusion: These results indicate that it is possible to classify patients using these parameters as prognostic indicators.

Key words: guidelines, hospital-acquired pneumonia, Japan, surveillance. 


\section{INTRODUCTION}

Hospital-acquired pneumonia (HAP) is the second most common nosocomial infection, and mortality due to HAP is the highest among hospital-acquired infections. ${ }^{1-3}$ Because drug-resistant pathogens are common causative organisms in compromised hosts, treatment is difficult and antibiotic selection is important. Delays in appropriate initial antimicrobial therapy have been associated with excess mortality due to HAP. ${ }^{4-8}$ In the United States (US), the management of HAP has been standardized by the publication of treatment guidelines by the American Thoracic Society (ATS)/Infectious Diseases Society of America (IDSA). ${ }^{1,2}$

The ATS guidelines define HAP occurring within the first 5 days of hospitalization as early-onset disease, and HAP occurring thereafter as late-onset disease. Due to differences between the health insurance systems of Japan and the US, however, patients are more likely to have near-normal immunity throughout long-term hospitalization in Japan. Thus, it would not be appropriate to apply the US treatment guidelines directly to the Japanese situation. ${ }^{9,10}$

To define the criteria relevant to the actual condition in Japan, guidelines for the management of HAP were published by the Japanese Respiratory Society (JRS) in March 2002. ${ }^{9}$ In these guidelines, patients were divided into groups I, II, III and IV, ranging from mild to severe. Following the publication of these guidelines, a nationwide multicentre collaborative survey was conducted to ascertain the clinical details of HAP across Japan, with the objective of investigating the appropriateness of the guidelines. ${ }^{9}$

The results of this survey suggested a need for revision of the classification of pneumonia, because there was a remarkable disparity in the numbers of patients in each group; more than $90 \%$ of patients were stratified into Groups III or IV, while only 4 patients $(0.3 \%)$ were stratified into Group I. In addition, among patients categorized in Group III, there were differences in mortality rate with the severity of pneumonia. ${ }^{10}$ 
The severity classification in the current JRS guidelines was based on clinical evaluation methods for new antimicrobial agents for treating respiratory infections, ${ }^{11}$ with emphasis being on evaluating the efficacy of investigational drugs. Taking into account the fact that the primary endpoint of pneumonia is the prognosis (mortality rate), it is more appropriate to select parameters that are indicators of prognosis, such as the CURB-65 (confusion, urea $>7 \mathrm{mmol} / \mathrm{L}$, respiratory rate $\geq 30 / \mathrm{min}$, low systolic $(<90 \mathrm{~mm} \mathrm{Hg}$ ) or diastolic ( $\leq 60 \mathrm{~mm} \mathrm{Hg}$ ) blood pressure, and aged $\geq 65$ years) ${ }^{12}$ and A-DROP (age, dehydration, respiratory failure, orientation disturbance, shock blood pressure) ${ }^{13}$ severity criteria for community-acquired pneumonia (CAP).

In this study, the factors affecting prognosis of HAP were examined, and those most relevant for severity classification of HAP in Japan were selected. 


\section{METHODS}

\section{Subjects}

The cases used in this study were identified in a nationwide multicentre collaborative survey of HAP in Japan. ${ }^{10}$ The survey was conducted during June 2002 - May 2004, and details of 1,356 patients from 254 hospitals were analyzed.

\section{Selection of primary assessment parameters}

For the primary assessment parameters, starting with the parameter with the most significant effect on prognosis, we selected the following factors as prognostic indicators: "malignant tumour or immunocompromised status", "impaired consciousness", "requiring fraction of inspired oxygen $\left(\mathrm{FiO}_{2}\right)>35 \%$ to maintain $\mathrm{SaO}_{2}>90 \%$ ", "male aged 70 years or older, or female aged 75 years or older" and "oliguria or dehydration". ${ }^{10}$ Of these factors, “malignant tumour" (odds ratio: 3.555; 95\% confidence interval [CI] 2.497-5.061), "impaired consciousness" (odds ratio: 2.406; 95\% CI 1.667-3.472), "requiring $\mathrm{FiO}_{2}>35 \%$ to maintain $\mathrm{SaO}_{2}>90 \%$ " (odds ratio: 1.567; 95\% CI 1.071-2.291), and "oliguria" (odds ratio: 2.863; 95\% CI 1.286-6.376) had significant effects on prognosis in the HAP survey. ${ }^{10}$

The survey showed that prognosis was poor for patients with cellular immunocompromised status, while some patients had multiple immunosuppressive states such as cellular and humoral immunosuppressive states. In addition, since there were no pronounced differences among the presumptive causative organisms (data not shown), these two conditions were merged as "immunocompromised status", and the assessment parameter was combined with the associated disease state, malignant tumour, to yield "malignant tumour and/or immunocompromised status".

Dehydration was not associated with prognosis in the HAP survey, but had an impact on clinical efficacy. ${ }^{10}$ Thus, it was combined with the associated disease state, oliguria, to 
create the assessment parameter "oliguria or dehydration", similar to the A-DROP criterion. ${ }^{13}$

\section{Selection of secondary assessment parameters}

Considering the possibility that patients with a poor prognosis may not be completely covered by the primary assessment parameters, we selected the following secondary assessment parameters as indicators of severity of pneumonia: "C-reactive protein (CRP) $\geq$ $200 \mathrm{mg} / \mathrm{L}$ " and "extent of infiltration on CXR covers at least $2 / 3$ of one lung". In the survey results, the factors "CRP $\geq 200 \mathrm{mg} / \mathrm{L}$ " (odds ratio: 1.322; 95\% CI 1.072-1.630) and "extent of infiltration on CXR covers at least $2 / 3$ of one lung" (odds ratio: $1.285 ; 95 \%$ CI 1.051-1.572) were found to affect prognosis. ${ }^{10}$

\section{Severity rating}

The severity rating was assessed using the criteria shown in Figure 1. Patients with a specified number or more of the primary risk factors were stratified into the high-risk group. Patients with any of the secondary risk factors were stratified into the moderate-risk group. All other patients were stratified into the low-risk group.

\section{Calculation of mortality rate according to severity, and statistical analysis}

Mortality rate was evaluated as all-cause death at 30 days after the start of the initial treatment, and the mortality rate was calculated according to severity. According to the IDSA/ATS HAP guidelines, the crude mortality rate for HAP may be as high as 30 to $70 \%{ }^{5}$ As the mortality rate for HAP in Japan is relatively low in comparison with the US, ${ }^{10} 30 \%$ was used as a yardstick for mortality rate in the high-risk group. Fisher's exact test was used for statistical analysis, and a risk rate less than 5\% was regarded as significant. 


\section{RESULTS}

\section{Characteristics of patients included in the HAP survey in Japan}

Details of the patient characteristics are summarized in Table 1. Males, patients aged 65 years or older, and patients with ventilator associated pneumonia accounted for $69.2 \%, 81.0 \%$, and $6.6 \%$ of the total, respectively. Early onset cases accounted for $9.2 \%$ of all cases, and cases with onset of symptoms more than 30 days after hospitalization accounted for $48.7 \%$. The most common pathogens were Staphylococcus aureus (including methicillin-resistant $S$. aureus [MRSA]) (25.5\%), followed by Pseudomonas aeruginosa (18.3\%) and Klebsiella pneumoniae (8.2\%). The 30-day mortality rate was $19.8 \%$.

\section{Investigation of severity rating}

Stratification by primary assessment parameter

The distribution of mortality rate by number of primary assessment parameters is shown in Table 2 . When the cases were stratified by a threshold value of " 3 factors", the mortality rate for the group with "3 or more factors" was $40.8 \%$, which was the closest to the target mortality rate $(30 \%)$, and higher than the mortality rate for " 2 or fewer factors" $(15.3 \%)$. In the following analysis, the high-risk group was defined as the patients with " 3 or more" of the primary risk factors.

\section{Threshold value for CRP}

The distribution of mortality rates by CRP value at diagnosis is shown in Table 3 . When the cases were stratified by a threshold value of $200 \mathrm{mg} / \mathrm{L}$, the mortality rate in the " $\geq$ $200 \mathrm{mg} / \mathrm{L}$ " group was $30.9 \%$, which was the closest to the target mortality rate $(30 \%)$, and higher than the mortality rate in the " $<200 \mathrm{mg} / \mathrm{L}$ " group $(17.7 \%)$. In the following analysis, severity was stratified based on a CRP value of $200 \mathrm{mg} / \mathrm{L}$. 


\section{Mortality rate by degree of severity}

The mortality rate in the moderate-risk group was $24.9 \%(69 / 277)$. This mortality rate was higher than that in the low-risk group $(12.1 \%, 101 / 834)$ (Table 4). Among the patients in the low-risk group, 194 (34.9\%) were assessed as severe under the current JRS HAP guidelines (Table 5). The mortality rate for these patients was 19.1\% (37/194).

\section{Comparison of the severity classification criteria evaluated in this study with CURB-65}

CURB-65 is a clinical prediction rule that has been validated for predicting mortality in CAP. $^{12}$ The performance of CURB-65 in predicting mortality due to HAP in Japan has been examined. ${ }^{10}$ As information on blood-urea nitrogen was not collected in the HAP survey, dehydration was used as a substitute. Patients with CURB-65 scores of $0-1,2$, and 3-5 accounted for $70.3 \%(953 / 1356), 20.4 \%(277 / 1356)$, and $9.3 \%(126 / 1356)$ of the total, respectively. In comparison with the percentage of patients in the low-risk group $(61.8 \%$, 838/1356), the percentage with CURB 0-2 was slightly higher. The mortality rate increased with increasing CURB-65 scores; the mortality rates of patients with CURB-65 scores of 0-1, 2, and 3-5 were $15.1 \%(143 / 950), 27.5 \%(76 / 276)$, and 39.2\% (49/125), respectively.

The mortality rate for patients classified by CURB-65 score was compared with that for patients classified according to the criteria used in this study (Table 6). Thirty-one patients in the high-risk group had CURB-65 scores of $0-1$, and the mortality rate was $45.2 \%$. In contrast, 15 patients with CURB-65 scores of 3-5 were categorized in the low-risk group, and the mortality rate for these patients was $13.3 \%$. 


\section{DISCUSSION}

Based on the results obtained in a nationwide survey, the classification of severity of HAP in the current guidelines was reviewed, and a new classification scheme based on prognostic indicators was evaluated.

A process of scoring individual risk factors and classifying severity on the basis of those scores, similar to the PORT (pneumonia outcomes research team) classification in the IDSA CAP guidelines, ${ }^{14}$ was initially considered. However, as the basic principle was to ensure that the JRS HAP guidelines would be widely used by non-specialized physicians in practical clinical settings, a classification scheme that required cumbersome calculation of scores would not have been appropriate. Therefore concise classification criteria, similar to the A-DROP criteria in the JRS CAP guidelines, ${ }^{13}$ were developed.

Risk factors associated with prognosis in the HAP survey, ${ }^{10}$ were selected and investigated, to identify those most relevant for severity classification. The five factors selected were very similar to the assessment factors used in A-DROP, except that "malignant tumour or immunocompromised status" was adopted instead of "blood pressure (shock)". For the assessment of severity in HAP, the underlying disease was more important than the physical condition of the patients. Although dehydration is a subjective parameter, A-DROP includes dehydration as a severity assessment parameter that is an indicator of prognosis. As the JRS CAP guidelines are widely used and accepted in clinical settings in Japan, dehydration was similarly adopted as a severity assessment parameter for HAP. The mortality rate for the group with " 3 or more factors" was $40.8 \%$. The mortality rate for patients with five primary assessment factors was probably relatively low because only seven patients were in this category.

As some patients with a poor prognosis may be classified as "mild" on the primary criteria alone, secondary criteria for assessment of the severity of pneumonia were also 
examined, and CRP and infiltrates on CXR were selected as the secondary parameters. It has been reported that there is no correlation between CRP and the prognosis of patients with CAP $;^{15,16}$ however, CRP was correlated with the prognosis of HAP patients. ${ }^{10}$ In addition, when the mortality rate was calculated for a threshold CRP value of $200 \mathrm{mg} / \mathrm{L}$, there was an appreciable difference in mortality rates between patients with high and low values for CRP, and it was therefore considered appropriate to include CRP as an assessment parameter.

With the classification based on the criteria used in this study (Fig. 1), the mortality rate in the high-risk group was $40.8 \%(98 / 240)$, which was significantly higher than the rates in the moderate and low-risk groups $[24.9 \%(69 / 277), P<0.001$ and $12.1 \%(101 / 834)$, $P<0.001$, respectively]. When this severity classification was compared with that using the CURB-65 criteria, the percentage of patients classified in the low-risk group was considerably higher using CURB-65. The low-risk group, as classified by CURB-65, contained a sub-group with a high mortality rate, and CURB-65 may underestimate the number of serious HAP cases in Japan. This is probably because CURB-65 does not use factors affecting HAP prognosis, such as malignant tumour, ${ }^{10}$ as evaluation parameters. Thus, these revised severity classification criteria can be more appropriately applied to HAP cases than CURB-65.

As the mortality rate in the high-risk group was high (40.8\%), it seems appropriate to recommend potent combination therapy (carbapenems or other $\beta$-lactams with anti-pseudomonal activity, plus aminoglycosides or fluoroquinolones, etc.) for this group of patients, in accordance with the antibiotic recommendations for groups at risk for multidrug resistant pathogens in the ATS/IDSA HAP guidelines. ${ }^{2}$ It would also be important to de-escalate the therapy whenever possible.

In contrast, for the low-risk group, excluding patients with risk factors for Pseudomonas aeruginosa pneumonia, including long-term hospitalization, prior use of third-generation cephalosporins, $\mathrm{COPD},{ }^{17-19}$ and aspiration pneumonia involving anaerobic 
organisms, ${ }^{20}$ it may be appropriate to recommend monotherapy (e.g., penicillins, third-generation cephems, etc.) rather than potent combination therapy as initial treatment.

In the ATS/IDSA HAP guidelines, if MRSA risk factors are present or recognized or if there is a high incidence locally, use of anti-MRSA drugs is recommended as initial empirical therapy. ${ }^{2}$ In a survey conducted in Japan, the incidence of S. aureus, principally MRSA, as the presumptive causative organism was high, but initial use of glycopeptide drugs was as low as $3.8 \% .{ }^{10}$ Considering the fact that both clinical efficacy and prognosis are poor in MRSA pneumonia, ${ }^{5}$ it may be necessary to use anti-MRSA drugs as initial therapy for patients at risk of infection with MRSA. As reported in previous studies, ${ }^{21-23}$ MRSA infection should be suspected on the basis of Gram-stained specimens or other evidence such as, 1) long-term antimicrobial treatment, 2) a history of long-term hospitalization, and 3) a history of MRSA infection or colonization. As the risk of MRSA infection is significant (data not shown), initial combination therapy that includes an anti-MRSA drug may lead to improved prognoses.

Using the severity classification criteria examined in this study, we were able to definitively classify patients into three groups based on prognostic indicators. However, the low-risk group included some patients assessed as severe using the current guidelines, and it is possible that severity in these cases was underestimated (Table 5). In these cases, escalation of antibiotic therapy may be necessary.

In conclusion, clinical parameters were examined and new severity classification criteria for HAP were established. Verification of the appropriateness of these revisions under clinical conditions will be important, and it will also be essential to periodically review the classification scheme with the aim of providing appropriate guidelines. 


\section{ACKNOWLEDGEMENT}

The authors thank Shunsuke Tani and Yasuhide Uchimura, Dainippon Sumitomo Pharma Co. Ltd., Osaka, Japan for their assistance with the statistical analyses. 


\section{REFERENCES}

1 American Thoracic Society. Hospital-acquired pneumonia in adults; diagnosis, assessment of severity, initial antimicrobial therapy, and preventative strategies. A consensus statement. Am. J. Respir. Crit. Care Med. 1996; 153: 1711-25.

2 American Thoracic Society/Infectious Diseases Society of America. Guidelines for the management of adults with hospital-acquired, ventilator-associated, and healthcareassociated pneumonia. Am. J. Respir. Crit. Care Med. 2005; 171: 388-416.

3 Craven DE, Steger KA. Epidemiology of nosocomial pneumonia: new perspectives on an old disease. Chest 1995; 108: S1-16.

4 Luna CM, Vujacich P, Niederman MS, Vay C, Gherardi C et al. Impact of BAL data on the therapy and outcome of ventilator-associated pneumonia. Chest 1997; 111: 676-85.

5 Kollef MH, Sherman G, Ward S, Fraser VJ. Inadequate antimicrobial treatment of infections: a risk factor for hospital mortality among critically ill patients. Chest 1999; 115: $462-74$.

6 Iregui M, Ward S, Sherman G, Fraser VJ, Kollef MH. Clinical importance of delays in the initiation of appropriate antibiotic treatment for ventilator-associated pneumonia. Chest 2002; 122: 262-8.

7 Ibrahim EH, Sherman G, Ward S, Fraser VJ, Kollef MH. The influence of inadequate antimicrobial treatment of bloodstream infections on patient outcomes in the ICU setting. Chest 2000; 118: 146-55.

8 Cosgrove SE, Kaye KS, Eliopoulous GM, Carmeli Y. Health and economic outcomes of the emergence of third-generation cephalosporin resistance in Enterobacter species. Arch. Intern. Med. 2002; 162: 185-90.

9 The committee for the Japanese Respiratory Society guidelines in the management of 
respiratory infections. The Japanese Respiratory Society guidelines for the management of hospital-acquired pneumonia in adults. Respirology 2004; 9 (Suppl.): S1-S59.

10 Watanabe A, Yanagihara K, Kohno S, Matsushima T, HAP study group. Multicenter survey on hospital-acquired pneumonia and the clinical efficacy of first-line antibiotics in Japan. Intern. Med. 2008; 47: 245-54.

11 Saito A, Miki F, Oizumi K, Rikitomi N, Watanabe A et al. Clinical evaluation methods for new antimicrobial agents to treat respiratory infections: Report of the Committee for the Respiratory System, Japan Society of Chemotherapy. J. Infect. Chemother. 1999; 5: 110-23.

12 Lim WS, van der Eerden MM, Laing R, Boersma WG, Karalus $\mathrm{N}$ et al. Defining community acquired pneumonia severity on presentation to hospital: an international derivation and validation study. Thorax 2003; 58: 377-82.

13 The committee for the Japanese Respiratory Society guidelines in the management of respiratory infections. The Japanese Respiratory Society guidelines for the management of community-acquired pneumonia in adults. Respirology 2006; 11: S79-S133.

14 Bartlett JG, Breiman RF, Mandell LA, File TM Jr. Community-acquired pneumonia in adults: guidelines for management. The Infectious Diseases Society of America. Clin. Infect. Dis. 1998; 26: 811-38.

15 Fine MJ, Auble TE, Yealy DM, Hanusa BH, Weissfeld LA et al. A prediction rule to identify low-risk patients with community-acquired pneumonia. N. Engl. J. Med. 1997; 336: $243-50$.

16 Hedlund J. Community-acquired pneumonia requiring hospitalisation. Factors of importance for the short-and long term prognosis. Scand. J. Infect. Dis. Suppl. 1995; 97: 1-60.

17 Rello J, Ausina V, Ricart M, Castella J, Prats G. Impact of previous antimicrobial therapy on the etiology and outcome of ventilator-associated pneumonia. Chest 1993; 104: 1230-5. 
18 Niederman MS. Gram-negative colonization of the respiratory tract: pathogenesis and clinical consequences. Semin. Respir. Infect. 1990; 5: 173-84.

19 Talon D, Mulin B, Rouget C, Bailly P, Thouverez M et al. Risks and routes for ventilator-associated pneumonia with Pseudomonas aeruginosa. Am. J. Respir. Crit. Care Med. 1998; 157: 978-84.

20 Marik PE, Careau P. The role of anaerobes in patients with ventilator-associated pneumonia and aspiration pneumonia: a prospective study. Chest 1999; 115: 178-83.

21 Iwahara T, Ichiyama S, Nada T, Shimokata K, Nakashima N. Clinical and epidemiologic investigations of nosocomial pulmonary infections caused by methicillin-resistant Staphylococcus aureus. Chest 1994; 105: 826-31.

22 Rello J, Torres A, Ricart M, Valles J, Gonzalez J et al. Ventilator-associated pneumonia by Staphylococcus aureus. Comparison of methicillin-resistant and methicillin-sensitive episodes. Am. J. Respir. Crit. Care Med. 1994; 150: 1545-9.

23 DeRyke CA, Lodise TP Jr, Rybak MJ, McKinnon PS. Epidemiology, treatment, and outcomes of nosocomial bacteremic Staphylococcus aureus pneumonia. Chest 2005; 128: $1414-22$. 


\section{FIGURE LEGENDS}

Figure 1. Flowchart depicting new procedure for assessment and classification of severity. Patients with hospital-acquired pneumonia (HAP) were assessed according to the criteria and treatment is determined by classification into one of the three risk groups. 
Table 1. Characteristics of patients with hospital-acquired pneumonia in a survey from Japan ${ }^{10}$

\begin{tabular}{|c|c|c|}
\hline Characteristic & Category & $\begin{array}{l}\text { Number of } \\
\text { patients (\%) }\end{array}$ \\
\hline Gender & male & $939(69.2 \%)$ \\
\hline Age (years) & 65 years or older & $1098(81.0 \%)$ \\
\hline \multirow[t]{2}{*}{ Onset time (days) } & $2 \leq<5$ & $125(9.2 \%)$ \\
\hline & $>30$ & $660(48.7 \%)$ \\
\hline Disease type & ventilator-associated pneumonia & $90(6.6 \%)$ \\
\hline \multirow[t]{3}{*}{ Presumed causative organism } & Staphylococcus aureus ${ }^{\mathrm{a}}$ & $208(25.5 \%)$ \\
\hline & Pseudomonas aeruginosa & $149(18.3 \%)$ \\
\hline & Klebsiella pneumoniae & $67(8.2 \%)$ \\
\hline
\end{tabular}

${ }^{\text {a }}$ Including methicillin-resistant S. aureus 
Table 2. Prognosis by the number of primary risk factors in the severity classification.

\begin{tabular}{|c|c|c|c|}
\hline Number of & \multicolumn{2}{|c|}{ Mortality rate ${ }^{\dagger}$} & \multirow[t]{2}{*}{$P$ value } \\
\hline 0 & $5.2 \%(6 / 115)$ & & \\
\hline 1 & $12.5 \%(70 / 561)$ & $15.3 \%$ & \multirow{5}{*}{$<0.001$} \\
\hline 2 & $21.6 \%(94 / 435)$ & $(170 / 1111)$ & \\
\hline 3 & $35.6 \%(62 / 174)$ & & \\
\hline 4 & $57.6 \%(34 / 59)$ & $40.8 \%$ & \\
\hline 5 & $-(2 / 7)$ & $(98 / 240)$ & \\
\hline
\end{tabular}

\section{*Fisher's exact test}

$\dagger$ Mortality rate was calculated by dividing the number of deaths by the number of patients for whom prognosis was assessed. 
Table 3. Prognosis by the concentration of C-reactive protein (CRP)

\begin{tabular}{|c|c|c|c|}
\hline $\mathrm{CRP}(\mathrm{mg} / \mathrm{L})$ & Mortality r & & $P$ value ${ }^{*}$ \\
\hline$<50$ & $14.6 \%(44 / 301)$ & \multirow{4}{*}{$\begin{array}{c}17.7 \% \\
(198 / 1119)\end{array}$} & \multirow{6}{*}{$<0.001$} \\
\hline$\geq 50,<100$ & $16.3 \%(59 / 361)$ & & \\
\hline$\geq 100,<150$ & $20.5 \%(53 / 259)$ & & \\
\hline$\geq 150,<200$ & $21.2 \%(42 / 198)$ & & \\
\hline$\geq 200,<250$ & $26.5 \%(30 / 113)$ & $30.9 \%$ & \\
\hline$\geq 250$ & $36.3 \%(33 / 91)$ & $(63 / 204)$ & \\
\hline
\end{tabular}

\footnotetext{
Fisher's exact test

$\dagger$ Mortality rate was calculated by dividing the number of deaths by the number of patients for whom prognosis was assessed.
} 
Table 4. Prognosis by severity using the revised severity classification criteria

\begin{tabular}{lccc}
\hline \multicolumn{1}{c}{ Category } & Cases & Mortality rate & $P^{\dagger}$ \\
\hline Low-risk & 838 & $12.1 \%(101 / 834)$ & $<0.001$ \\
& & & \\
\hline Moderate-risk & 277 & $24.9 \%(69 / 277)$ & \\
\hline High-risk & 241 & $40.8 \%(98 / 240)$ & \\
\hline
\end{tabular}

*Fisher's exact test

$\dagger$ Mortality rate was calculated by dividing the number of deaths by the number of patients for whom prognosis was assessed. 
Table 5. Correlation between severity classification as assessed using the revised guidelines and the current guidelines

\begin{tabular}{|c|c|c|}
\hline \multirow[t]{2}{*}{ Category } & \multicolumn{2}{|c|}{ Mortality rate by severity using the current guidelines* } \\
\hline & Mild or moderate cases & Severe cases \\
\hline Low-risk & $10.0 \%(64 / 640)$ & $19.1 \%(37 / 194)$ \\
\hline Moderate-risk & $16.5 \%(23 / 139)$ & $33.3 \%(46 / 138)$ \\
\hline High-risk & $16.7 \%(3 / 18)$ & $39.2 \%(95 / 222)$ \\
\hline
\end{tabular}

*Mortality rate was calculated by dividing the number of deaths by the number of patients for whom prognosis was assessed. 
Table 6. Correlation between severity classification using the revised severity rating criteria and mortality rates for cases classified according to the CURB-65 criteria.

\begin{tabular}{|c|c|c|c|}
\hline \multirow[t]{2}{*}{ Category } & \multicolumn{3}{|c|}{ Mortality rate according to CURB-65 scores* } \\
\hline & $0-1$ & 2 & $3-5$ \\
\hline Low-risk & $10.9 \%(78 / 713)$ & $19.8 \%(21 / 106)$ & $13.3 \%(2 / 15)$ \\
\hline Moderate-risk & $24.8 \%(51 / 206)$ & $21.9 \%(14 / 64)$ & $-(4 / 7)$ \\
\hline High-risk & $45.2 \%(14 / 31)$ & $38.7 \%(41 / 106)$ & $41.7 \%(43 / 103)$ \\
\hline
\end{tabular}

*Mortality rate was calculated by dividing the number of deaths by the number of patients for whom prognosis was assessed.

CURB-65, confusion, urea $>7 \mathrm{mmol} / \mathrm{L}$, respiratory rate $\geq 30 / \mathrm{min}$, low systolic $(<90 \mathrm{~mm}$ $\mathrm{Hg}$ ) or diastolic ( $\leq 60 \mathrm{~mm} \mathrm{Hg}$ ) blood pressure, and aged $\geq 65$ years. 
Figure 1

Primary step
(1) Malignant tumour or immunocompromised status
(2) Impaired consciousness
(3) Requiring $\mathrm{FiO}_{2}>35 \%$ to maintain $\mathrm{SaO}_{2}>90 \%$
(4) Male ( $\geq 70$ years)、 Female $(\geq 75$ years)
(5) Oliguria or dehydration

Less than 2 risk factors

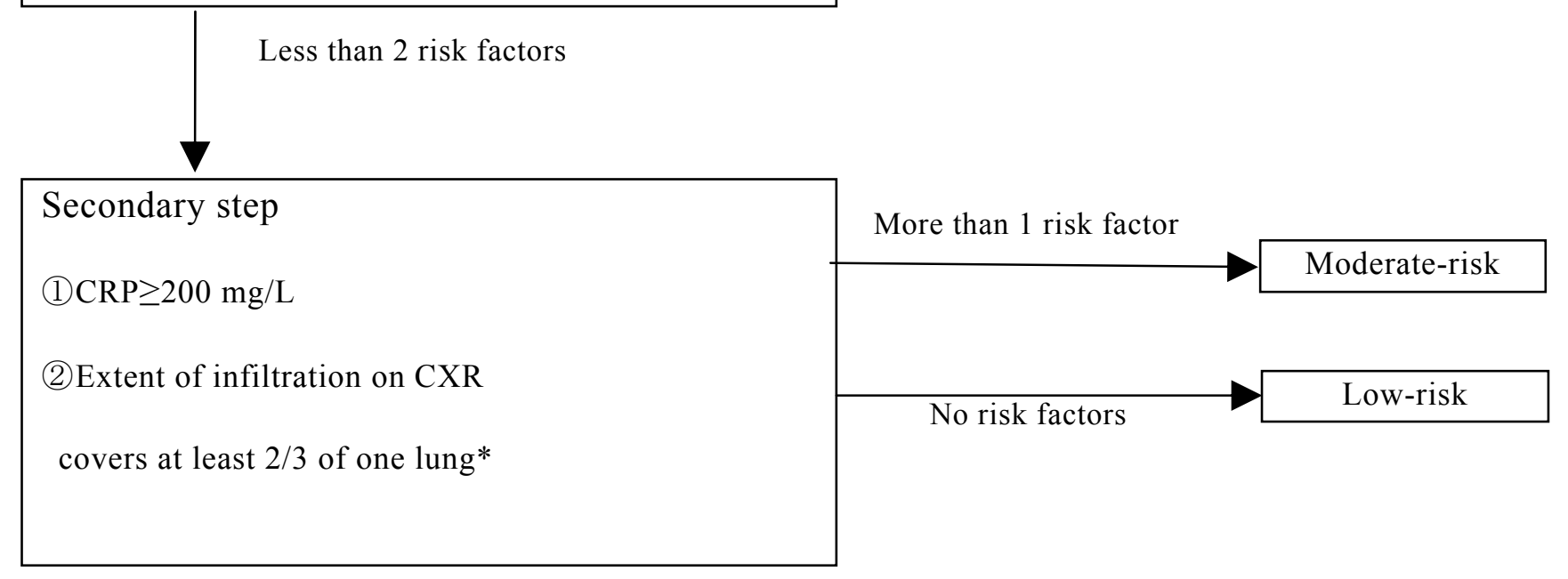

* If there is more than one area of infiltration, the rating is determined by the sum of all the lesions 